Muséologies

Les cahiers d'études supérieures

muséologies

\title{
Regards croisés sur les mondes de l'autoportrait : le cas des artistes d'origine marocaine Hicham Benohoud, Mehdi-Georges Lahlou et Zakaria Ramhani
}

\section{Alexia Pinto Ferretti}

Volume 9, numéro 1, 2018

Monde des Arts / Arts des mondes

URI : https://id.erudit.org/iderudit/1052628ar

DOI : https://doi.org/10.7202/1052628ar

Aller au sommaire du numéro

Éditeur(s)

Association Québécoise de Promotion des Recherches Étudiantes en Muséologie (AQPREM)

ISSN

1718-5181 (imprimé)

1929-7815 (numérique)

Découvrir la revue

Citer cet article

Pinto Ferretti, A. (2018). Regards croisés sur les mondes de l'autoportrait : le cas des artistes d'origine marocaine Hicham Benohoud, Mehdi-Georges Lahlou et Zakaria Ramhani. Muséologies, 9(1), 61-78. https://doi.org/10.7202/1052628ar

\section{Résumé de l'article}

This article takes a contemporary look at the practice of self-portraiture by three artists from Morocco: Hicham Benohoud, Mehdi-Georges Lahlou and Zakaria Ramhani. The career trajectories of these creators illustrate the various dynamics of institutional opposition and integration mechanisms that belong to the new geographies of art, in the era of globalization. The article's goal is to investigate the manner in which individual, local and global concerns are expressed in their understanding of an imaginary projection of corporality. The works are studied through the lens of local characteristics particular to the artist's Maghreb-Muslim culture. In the first section, the artists' careers, in relation to their training and the places where they have exhibited their work, are presented in tandem with the paradoxes of globalization. It is thus specified that if the artists were trained primarily in the West or made their careers there to achieve international success, it can be attributed to a lack of support from the Moroccan artistic scene, but also to the difficult access to the international art world for artists coming from the art scenes of the Maghreb. In the second part, we postulate that the artists' self-portraits attest to the different issues related to Maghreb-Muslim culture, such as the current socio-political situation in Morocco and the Islamic ban on representation. Lastly, we maintain that these self-portraits are a wider reflection of the varied concerns that transcend the borders of the Maghreb, as the artists reflect on the meeting of the Eastern and Western worlds.
Tous droits réservés (C) Association Québécoise de Promotion des Recherches Étudiantes en Muséologie (AQPREM), 2018
Ce document est protégé par la loi sur le droit d'auteur. L'utilisation des services d'Érudit (y compris la reproduction) est assujettie à sa politique d'utilisation que vous pouvez consulter en ligne.

https://apropos.erudit.org/fr/usagers/politique-dutilisation/ 
Article trois

Regards croisés sur les mondes de l'autoportrait: le cas des artistes

d'origine marocaine Hicham Benohoud, Mehdi-Georges Lahlou et Zakaria

Ramhani

Alexia Pinto Ferretti 
Détentrice d'une maîtrise en histoire de l'art (2015, UQAM), Alexia Pinto Ferretti est candidate au doctorat interuniversitaire en histoire de l'art à l'Université de Montréal. Subventionnées par le FRQSC, ses recherches doctorales portent sur les stratégies d'autoreprésentation des identités autochtones dans le travail d'artistes visuels dont la démarche s'inscrit en rapport au cyberespace. Elle a publié des articles sur des artistes des communautés culturelles ou autochtones dans les revues $R A C A R$, Espace art actuel et Esse art + opinions. 
Présentée en 2015 au Musée des Beaux-arts de Montréal, l'exposition Benjamin-Constant: Merveilles et mirages de l'orientalisme proposait au public une réflexion plurielle sur la mise en scène de l'imaginaire d'un Orient mystérieux et intemporel sous le regard de l'Occident. Figure phare de l'orientalisme au $19^{\mathrm{e}}$ siècle, BenjaminConstant (1845-1902) est particulièrement reconnu pour avoir illustré la Médina de Tanger, lors de ses différents séjours au Maroc (1870, 1871-1873), à travers des œuvres monumentales s'inscrivant tant dans une mouvance ethnographique que romantique. L'exposition n'évacuait pas les essentielles critiques des auteurs du post-colonialisme, comme Edward Said, sur le mouvement orientaliste en tant que vision idéalisée d'un Orient immobile dans le temps. Dans le catalogue de l'exposition, l'historienne de l'art Christine Peltre explique avec justesse que les représentations de Benjamin-Constant illustrent un Orient intemporel et imperméable aux changements sociétaux et technologiques ${ }^{1}$. Par exemple, dans les représentations des harems, les odalisques sont dépeintes dans des positions passives, en attente, donnant ainsi l'impression qu'elles sont figées dans le passé. Devant la nécessité de remettre en question ces représentations stéréotypées de l'Orient, les commissaires ont aussi donné une place au travail de trois artistes contemporaines d'origine marocaine: Yasmina Bouziane, Lalla Essaydi et Majida Khattari. À travers une démarche photographique, ces femmes marocaines, anciens modèles par excellence du regard orientaliste, deviennent des agents actifs en reprenant possession de leurs représentations à travers différentes stratégies de performativité du Soi. Elles mettent en récit la complexité des identités contemporaines maghrébines en cette époque où la mondialisation crée de nouvelles frontières du Soi et

1 PELTRE Christine. «Un orientalisme de Batignolles? ". Benjamin-Constant: merveilles et mirages de l'orientalisme, (BLONDIL Nathalie dir.), Musée des Beaux-arts de Montréal et Musée des Augustins: Hazan, 2015, p. 103-112.

2 L'article se base sur le mémoire de maîtrise en histoire de l'art que l'auteure a réalisé en 2015 à l'Université du Québec à Montréal, qui s'intitule: « Visage global: l'altération identitaire face à la question de la globalisation culturelle dans le travail de huit artistes d'origines culturelles différentes ". où plusieurs artistes vivent dans des espaces interstitiels entre l'Orient et l'Occident. La scénographie de l'exposition, où les œuvres de Benjamin Constant côtoient celles des trois artistes marocaines, crée ainsi un face-à-face temporel où une vision passéiste des figures arabes est remise en mouvement à travers des œuvres qui font écho aux défis actuels auxquels sont confrontés les artistes maghrébins qui évoluent dans un monde de l'art pluriel, entre Orient et Occident.

Visant à approfondir la question des démarches d'autoreprésentation du Soi propres à notre contemporanéité, cet article aborde les autoportraits de trois artistes actuels d'origine marocaine qui évoluent entre différents mondes des arts: Hicham Benohoud, Mehdi-Georges Lahlou et Zakaria Ramhani ${ }^{2}$. L'objectif est d'interroger la manière dont les trois artistes expriment, à travers l'autoportrait, une vision des identités culturelles à la conjonction des différents mondes : individuel, local et global. Il est soutenu qu'à travers leurs expériences du corps, ces artistes traduisent différents problèmes liés à des spécificités locales, propres à la culture maghrébo-musulmane. Cependant, ces artistes représentent aussi leurs expériences professionnelles de la mobilité et de la rencontre avec l'Autre, en cette époque de " perte du contexte $^{3}$ ". En effet, des sociologues comme Raymonde Moulin ont expliqué que les rencontres associées au nomadisme chez les artistes contemporains « [...] sont à l'origine d'espaces intermédiaires entre les scènes artistiques locales et le monde de l'art international ${ }^{4}$ ". Étant donné que la mondialisation peut être un véhicule d'homogénéisation des identités culturelles, on accordera la priorité aux spécificités socioculturelles du pays ou de la région d'où proviennent les trois artistes contemporains

3 BELTING Hans et Andrea BUDDENSIEG (dir). The Global Art World. Audiences, Marketsand Museums, Ostfildern: Hatje Cantz Verlag, 2009, p. 15.

4 MOULIN Raymonde. Le marché de l'art: mondialisation et nouvelles technologies, Éditions Flammarion: Paris, 2003, p. 76 . 
dans l'appréhension des œuvres. Il sera aussi pris en compte la question des interactions sur le plan local et global, qui marque leurs parcours professionnels. Mentionnons que les anthropologues Nicholas Thomas et Diane Losche ont développé dans cette perspective la notion de " double vision " pour expliquer la nécessité de considérer les relations entre le processus de mondialisation et les trajectoires artistiques spécifiques de chaque région ${ }^{5}$. Selon l'historien de l'art Thomas Fillitz, les deux auteurs, à travers cette double vision, encouragent les artistes et les universitaires à " [...] consider the global art world as a disjunctive order, to look at the production of contemporary art from the perspective of dislocated centers, and to acknowledge specific historical trajectories of conjuncture and disjuncture between local art worlds and the global art world $^{6}$ ». Cette approche méthodologique est indispensable lorsqu'on aborde la production artistique des pays de l'Afrique et, particulièrement, ceux du Maghreb qui sont historiquement des carrefours d'échanges et de tensions entre les différentes civilisations. Dans cette perspective, les démarches des artistes permettent de réfléchir aux différentes dynamiques institutionnelles en termes d'opposition et d'intégration propres aux nouvelles géographies de l'art à l'époque de la mondialisation. La situation des artistes met ainsi en exergue l'influence encore présente de l'époque coloniale sur le réseau institutionnel culturel marocain qui se développe principalement à travers un réseau public de musées ethnographiques ou célébrant l'indépendance, marquant ainsi une double déconnexion des préoccupations locales de la scène artistique et de la population marocaine.

5 Voir à ce sujet: LOSCHE Diane et Nicholas THOMAS. Double vision: art histories and colonial histories in the Pacific, Cambridge University Press : Cambridge (MA), 1999, 304 p.

\section{Être un artiste marocain au temps de la mondialisation: une dynamique duelle}

Depuis la fin de la guerre froide, les mondes de l'art occidental se démocratisent graduellement : l'apparition de résidences internationales, de biennales et de foires d'art sont les signes multiples d'une extension géographique de l'offre et de la demande. Dans ce contexte de déterritorialisation où les frontières économiques et culturelles s'estompent, les artistes sont de plus en plus nomades dans leurs choix d'institutions de formation et des lieux où ils exposent ${ }^{7}$. Hicham Benohoud, Mehdi-Georges Lahlou et Zakaria Ramhani sont justement des artistes dont le parcours professionnel traverse les océans. D'abord, Hicham Benohoud (1968) est diplômé des Beaux-Arts à l'Institut de Marrakech et de l'École supérieure des Arts décoratifs de Strasbourg. Il vit principalement au Maroc, mais il est représenté par la Galerie VU à Paris et la Galerie 21 à Casablanca, et fait régulièrement des résidences d'artistes en Europe depuis 1999. Mehdi-Georges Lahlou est né aux Sables-d'Olonne (1983) en France d'un père marocain et d'une mère espagnole. Diplômé de l'École régionale de Nantes Métropoles et de la Breda aux Pays-Bas, il crée des œuvres entre Paris, Bruxelles et Chicago. Il est présentement représenté par la Galerie Transit (Mechelen) et la Galerie Rabouan Moussion (Paris). Si ses œuvres ont été très peu présentées au Maghreb, la revue Diptyk dédiée à l'art contemporain marocain lui a consacré plusieurs articles dans les dernières années. Notre troisième artiste, Zakaria Ramhani, laisse son empreinte dans l'art contemporain international de Tanger, sa ville natale, à Montréal, sa ville d'adoption. En 2010, il a été nommé par le site Internet de référence Artprice comme l'un des 10 meilleurs artistes au monde de moins de 30 ans. Particulièrement présent dans le circuit de l'art international, il est actuellement représenté par la Galerie 21 à Casablanca et par la Galerie ARTSPACE à Dubaï.

6 FILLITZ Thomas. "Global art and Anthropology: the situated gaze and the local art worlds in Africa ". Dans BELTING Hans, BUDDENSIEG Andra et Peter Weibel (dir.). The Global Contemporary and The Rise of New Art Worlds. Londres: The MIT PRESS, 2013, p. 223.

7 MOULIN Raymonde, Op.cit., p. 78. 
L'expérience diversifiée de ces artistes des mondes de l'art visuel illustre différents enjeux propres aux phénomènes de la mondialisation qui peut être considérée tant comme à la source d'un conflit de civilisations que comme un vecteur positif de dialogue entre les cultures ${ }^{8}$. Une des principales critiques faites à la mondialisation est que sous le chapeau de la diversité culturelle, la biennalisation des mondes de l'art obligerait les artistes venus d'ailleurs ou originaires de différentes diasporas à s'adapter à un parcours institutionnalisé ponctué de centres de formation, de foires et de biennales appartenant toujours à " un centre " dont les limites de visibilité sont encore aujourd'hui définies par le monde occidental ${ }^{9}$. Déjà en 1978 , Howard Becker avait souligné l'existence, dans les mondes de l'art, de difficultés d'accès et de hiérarchisations sociales qui désavantagent les artistes en situation de minorité en raison de leur genre ou de leurs origines ethniques ${ }^{10}$. Silvia Naef, spécialiste d'art moderne arabe, explique ainsi que malgré les avancées amenées par les études postcoloniales pour pluraliser le concept de modernité, l'inclusion de la production locale des pays non occidentaux dans la sphère internationale est encore reléguée à la périphérie de l'histoire de l'art. Par exemple, les artistes originaires du Maghreb ou du MoyenOrient restent absents des grandes expositions internationales récentes visant à faire une rétrospective de la pratique de l'autoportrait depuis cinq siècles ${ }^{11}$. Il faut aussi mentionner que les artistes n'étant pas originaires d'un pays occidental ne connaissent souvent un succès interna-

8 WEIBEL Peter. "Globalization and Contemporary art ". Dans BELTING Hans, BUDDENSIEG Andrea et Peter Weibel (dir.). The Global Contemporary and The Rise of New Art Worlds, Londres: The MIT PRESS, 2013, p. 20-31.

9 Voir à ce sujet: TURGEON Laurier. « Les mots pour dire les métissages - Jeux et enjeux d'un lexique ". Dans Le soi et l'autre: l'énonciation de l'identité dans les contextes interculturels, (OUELLET Pierre dir), CÉLAT, Presses de l'Université Laval, Collections Intercultures, 2003, p. 383-402. 10 BUSCATTO Marie. "Quoi de neuf chez les artistes? Les " mondes de l'art " à l'épreuve du travail artistique ». Dans Les mondes pluriels de Howard S. Becker, (PERRENOUD, Marc dir.), Paris: Éditions La Découverte, 2013, p. 112. 11 Dans la dernière décennie, plusieurs grandes expositions dans différentes institutions muséales ont abordé le thème de l'autoportrait. Mentionnons Self-portrait: Renaissance to contemporary (2004) ayant eu lieu à la National Portrait Gallery de Londres, l'exposition Moi: autoportrait du xx $x^{e}$ siècle tional que lorsqu'ils sont représentés par une galerie réputée en Amérique du Nord ou en Europe $^{12}$. Précisons que si Mehdi-Georges Lahlou est soutenu par des galeries en Europe et aux États-Unis, Zakaria Ramhani et Hicham Benohoud doivent notamment leurs succès à la Galerie 21, la galerie la plus réputée du Maroc dont la mission est d'exposer le travail des artistes à succès de la diaspora marocaine, qui proposent des démarches originales.

Finalement, un dernier paradoxe caractérise la situation de ces trois artistes. En effet, bien que la scène artistique marocaine soit la plus dynamique au Maghreb, le pays est marqué par un désert structurel. Dans un vigoureux éditorial intitulé « L'art contemporain au Maroc, une situation à géométrie variable $\mathrm{e}^{13}$ ", Diptyk, la plus importante revue d'art contemporain du Maroc, mettait en lumière, en 2014, les contradictions propres au champ de l'art du pays. L'éditorial dénonçait notamment que malgré un succès à l'international, principalement pour des artistes appartenant à la diaspora marocaine, le milieu de l'art du Maroc souffre d'un manque flagrant d'investissement gouvernemental. Pour mieux comprendre cette critique, il est essentiel de faire ici une parenthèse historique pour expliquer les enjeux entourant les valeurs patrimoniales propres au contexte marocain. En effet, la genèse des musées au Maroc s'ancre dans l'histoire coloniale qui favorisera, à partir de la seconde moitié du $19^{\mathrm{e}}$ siècle, la constitution d'un réseau d'institutions d'expositions ethnographiques.

(2005) présentée au Musée du Luxembourg et au Palazzo Strozzi, et Autoportraits, de Rembrandt au selfie (2016), réalisée en partenariat avec la Staaliche Kunsthalle de Karlsruhe, la National Galleries of Scotland à Edimbourg et le Musée des Beaux-arts de Lyon. Ces expositions étaient conçues pour illustrer la pratique de l'autoportrait à travers l'histoire en valorisant notamment les œuvres déjà présentes dans les collections des musées participants. Malgré tout, plusieurs œuvres contemporaines étaient aussi exposées, posant ainsi un regard actuel sur la pratique de l'autoportrait jusqu'à la fin du 20e siècle. À ce titre, les autoportraits des artistes Shirin Neshat ou Rasheed Araeen, largement reconnus par le champ des arts, auraient pu avoir une place bien méritée.

12 MOULIN Raymonde, Op.cit., p. 78.

13 Rédaction de la revue Diptyk, « L'art contemporain au Maroc, une situation à géométrie variable ", revue Artabsolument et revue Diptyk, numéro spécial « L'art contemporain au Maroc ", mars 2014, p. 18-23. 
Fondées majoritairement sous le protectorat français (1915-1947), les collections des musées ethnographiques et archéologiques marocains étaient composées d'objets artisanaux qui illustraient le folklore local. Au même titre que l'orientalisme a contribué à diffuser des représentations passéistes des populations arabes, ces musées mettaient en exergue uniquement le caractère traditionnel de la société marocaine, justifiant ainsi la supériorité, voire la nécessité, de l'occupation du territoire par les Français, unique porteur de la modernité ${ }^{14}$. À l'aune de l'indépendance du pays (1956), le Maroc a hérité de ces musées ethnographiques, mais ne les a pas nécessairement actualisés en considérant les nouveaux idéaux modernes nationaux. En terme de patrimonialisation, c'est sous l'impulsion de la royauté marocaine que le pays a plutôt misé massivement sur la création d'un réseau public de musées mémoriels célébrant la lutte pour l'indépendance, voulant ainsi créer un sentiment de cohésion sociale favorisant la construction nationale ${ }^{15}$. Depuis plus de 20 ans, la mission d'exposer l'art moderne et contemporain marocain n'est donc pas menée par le gouvernement ou la royauté, mais est plutôt chapeautée par des instituts étrangers ou par le mécénat privé qui finance la création de musées et d'évènements d'envergure internationale, telle la biennale d'art de Marrakech (six présentations) ${ }^{16}$. Malgré des projets prometteurs, par exemple, les Villas des arts de Rabat et Casablanca et les Abattoirs de Casablanca, le public local tarde cependant à répondre présent, la majorité des Marocains fréquentant très peu les musées, considérant que ces derniers sont plutôt dédiés à l'élite et aux touristes. Le manque d'appui

14 Au sujet de la question muséale au Maroc, et plus largement au Maghreb, mentionnons l'ouvrage suivant: JELIDI Charlotte. Les musées au Maghreb et leurs publics: Algérie,

Maroc, Tunisie, Paris: La documentation française, 2013.

15 Ces musées de la Résistance et de l'armée de libération constituent présentement $20 \%$ du réseau muséal marocain.

16 À titre d'exemple, mentionnons la création du Musée de Marrakech (Fondation Omar Benjelloun) les Villas des Arts de Casablanca et Rabat (Fondation Omnium Nord Africain) et le Musée de la monnaie (Banque Al-Maghrib).

17 BHABHA Homi. Les lieux de la culture: Une théorie postcoloniale. Paris: Payot, 2007. (1994), p. 14. gouvernemental à la mise en valeur de l'art national, l'hégémonie du secteur privé en termes de financement et un public peu conscientisé par rapport aux œuvres contemporaines sont autant de barrières pour la diffusion et la réception du travail des artistes actuels. Plusieurs artistes, comme c'est le cas de Hicham Benohoud, Mehdi-Georges Lahlou et Zakaria Ramhani, s'orientent ainsi vers l'Europe et l'Amérique du Nord pour leurs formations et exposent majoritairement hors du Maroc, dans les différents circuits de biennales et de foires internationales.

Malgré des mondes de l'art qui évoluent à différentes vitesses, la mondialisation peut aussi être appréhendée comme un vecteur d'hétérogénéisation culturelle favorisant l'inclusion de zones géographiques jusqu'à récemment périphérique dans les circuits de l'art international. Par exemple, depuis 1989, plusieurs expositions d'art contemporain mondial permettent une plus grande visibilité d'artistes de différentes cultures. Les mondes de l'art se développent ainsi également selon un processus d'ouverture " sous le signe de l'égalité dans la différence ${ }^{17}$ ", particulièrement dans les grandes villes multiculturelles. Dans le texte incontournable « Un cosmopolitisme vernaculaire ", Homi Bhabha lance justement un appel à la valorisation d'un type de cosmopolitisme mené par et pour les peuples minoritaires et s'adaptant à différentes situations régionales et locales pour aller " au-delà du colonialisme ${ }^{18}$ ". Dans une perspective similaire, l'historien de l'art Terry Smith affirme que l'histoire de l'art des pays ayant connu une période coloniale, après différentes phases, s'inscrit actuellement dans une

18 Dans l'introduction des Lieux de la Culture (1994), Bhabha affirme que le terme post-colonialisme ne signifie pas " après le colonialisme ", mais " au-delà du colonialisme ". L'auteur ne s'intéresse donc pas aux suites de la colonisation, mais pose plutôt son attention vers un au-delà libérateur où les logiques du colonisateur n'auraient plus de pouvoir politique. 
recherche élargie d'un cosmopolitisme s'orientant vers des valeurs transnationales ${ }^{19}$. L'artiste Zakaria Ramhani explique justement que son identité culturelle est multiple : bien qu'elle s'ancre dans les lieux où il a grandi au Maroc, les grandes villes cosmopolites illustrent les différentes influences propres à son sentiment d'appartenance qui navigue entre des perspectives universelles et locales ${ }^{20}$.

Pour l'artiste et commissaire Peter Weibel, cette rencontre des civilisations s'avère aussi l'occasion d'une réécriture de l'histoire qui permettrait aux nouveaux mondes de l'art contemporain de s'éloigner des traditionnelles dynamiques occidentales d'inclusion et d'exclusion $^{21}$. En effet, les études postcoloniales valorisent une réévaluation multiple de l'histoire non pas sous la loupe occidentale, mais plutôt à travers l'angle des transferts culturels. L'auteure maorie Linda Tuhiwai Smith, une figure incontournable dans le cadre des méthodologies de décolonisation autochtone, explique que l'époque actuelle s'avère ainsi propice à un processus de " rewriting ", mais aussi de " rerighting $^{22} »$. Par ce jeu de mots particulièrement signifiant, l'auteure met de l'avant la nécessité pour les minorités culturelles et autochtones de reprendre le contrôle de leur histoire, écrite majoritairement par des étrangers, pour l'inscrire plutôt en harmonie avec leurs épistémologies.

\section{De nouvelles narrativités sous tension}

L'autoportrait est un genre artistique qui traverse l'histoire de l'art et qui se transforme continuellement selon le contexte socioculturel propre à l'époque où l'artiste évolue. D'un point de vue ontologique, il fait consensus dans la littérature scientifique que l'autoportrait dans sa définition la plus basique est le portrait de l'artiste « [...] et plus précisément son visage, c'est-à-dire la partie du corps par laquelle se manifeste son identité, visage auquel peuvent s'ajouter le buste, les mains et tout le reste du $\operatorname{corps}^{23}{ }^{2}$. Depuis le début du $20^{\mathrm{e}}$ siècle, les artistes créent des autoportraits de type introspectif pour exprimer leur état d'esprit, prendre position par rapport à différentes situations sociales et politiques, mais aussi pour aborder des enjeux contemporains, tel le rapport à l'Autre $^{24}$. Dans les années 1980, des artistes, tels Rasheed Araeen ou Jean-Michel Basquiat, ont utilisé la pratique de l'autoportrait pour illustrer que les discours de l'art fragmentent les expressions culturelles des artistes qui ne sont pas d'origine occidentale, mais aussi pour témoigner de leur expérience de la violence et de l'exclusion raciale. Les autoportraits de ces deux artistes étaient ainsi un appel à l'inclusion de nouvelles narrativités ne témoignant plus uniquement d'une vision occidentale des identités dans le panorama visuel mondial. Après les œuvres phares de Araeen et de Basquiat, mais aussi grâce à celles de Frida Kahlo et Shirin Neshat, la question de la représentation de nouvelles narrativités culturelles ne cesse d'être mise sous tension, notamment par le biais des études de genres et des études postcoloniales. Tel qu'il a été vu dans l'exposition BenjaminConstant: merveilles et mirages de l'orientalisme, l'autoportrait est un vecteur pour déconstruire l'imaginaire occidental qui fige l'Autre dans des représentations passéistes. Ainsi, le travail des
19 Selon Terry Smith, cette troisième phase a été précédée par une période où les artistes des pays ex-colonisés créaient généralement un type d'art en réaction à l'impérialisme occidental dans une optique de recentrement national. La deuxième phase abordait les questions identitaires en rapport à une vision simpliste de l'internationalisme. Voir à ce sujet : SMITH Terry. "Contemporary Art: World currents in Transition beyond Globalization ". Dans BELTING Hans, BUDDENSIEG Andra et Peter Weibel (dir.). The Global Contemporary and The Rise of New Art Worlds. Londres: The MIT PRESS, 2013, p. 188.

20 Cette information est tirée d'une entrevue que j'ai réalisée avec l'artiste, le $1^{\text {er }}$ juillet 2016.
21 WEIBEL, Op.cit., p. 27.

22 TUHIWAI SMITH Linda. Decolonizing Methodologies: Research and Indigenous Peoples, Londres: Zed Books, 2012 (1999), p. 39.

23 VAISSE Pierre. "Considérations intempestives sur l'autoportrait ". Dans PACCOUD Stéphane et Sylvie RAMOND (dir.). Autoportraits, de Rembrandt au selfie, Paris: Snoeck, 2016, p. 23. 24 MOULIN Joelle. L'autoportrait au $x x^{e}$ siècle, Paris: Adam Biro, 1999, p. 124. 
trois artistes à l'étude dans cet article exprime différentes zones de tensions propres à la pluralité du contexte de la mondialisation à travers une démarche artistique où la représentation du Soi devient une plateforme pour réfléchir à différents enjeux sociaux et politiques. Ces œuvres expriment une vision introspective de l'autoportrait en abordant différents éléments de la culture maghrébo-musulmane, tel l'interdit de la représentation, mais sont aussi le témoignage des expériences des artistes qui évoluent entre l'Orient et l'Occident.

\section{Hicham Benohoud: la blessure sociale du Soi}

Depuis le début des années 2000, Hicham Benohoud crée principalement des autoportraits où il défigure son visage pour mettre en scène la faiblesse du corps. Se représentant toujours de façon frontale et impassible, Benohoud exprime dans ses œuvres plusieurs de ses préoccupations envers la société marocaine actuelle.

Tout d'abord, la série Version Soft a été réalisée lors d'une résidence d'artiste à Bruxelles en 2002. Voulant initialement créer des photographies autour de la thématique « un musulman à Bruxelles ", Benohoud crée finalement une série d'autoportraits où son corps devient un espace d'expression et de protestation publique. Dans cette série de photographies, différents objets de petite taille, tels des clous, des pastilles ou des journaux, sont intégrés sur sa figure. Ces objets qui semblent cadenasser, voire blesser son visage, symbolisent la souffrance de l'artiste devant les enjeux socioculturels qui touchent le Maroc. Dans l'œuvre Sans-titre 3, le crâne de Benohoud, véritable motif iconique que l'artiste travaille de manière sérielle, est caché cette fois-ci par des trous béants colorés

25 BUDDENSIEG Andrea. «Rasheed Araeen: Artist and Critic ». Dans BELTING Hans, BUDDENSIEG Andra et Peter Weibel (dir.). The Global Contemporary and The Rise of New Art Worlds. Londres: The MIT PRESS, 2013, p. 45. 26 Voir à ce sujet: TEICHER Gaël. "Chronique d'une disparition ». Dans site Web de Hicham Benohoud. [En ligne], (2010) <http://www.hichambenohoud.com/benohoud/ index.php?option $=$ com_content\&view $=$ article $\&$ id $=59 \&$ Itemid=29>, consulté le 10 avril 2017. rappelant une blessure sanguinolente. Ces œuvres peuvent être mises en parallèle avec la série d'autoportraits intitulée 4 Ethnics drawings (1982), par Rashhed Araeen, dont les graffitis sur le visage de l'artiste font penser à des actes d'agression. Selon l'historien de l'art Andrea Buddensieg, ces autoportraits qui semblent avoir été attaqués par une foule invisible symbolisent les sentiments d'Araeen par rapport à son exclusion du monde artistique occidental ${ }^{25}$. Chez Benohoud, ces mises en scène transgressives correspondent à une stratégie pour exprimer son malaise envers les dogmatismes religieux, le conformisme social et la liberté d'expression qui reste aléatoire au Maroc. Benohoud exprime ses inquiétudes par une volonté de subversion. Il se distancie, par exemple, de l'interdit de la représentation du corps, particulièrement du visage, associé à la culture arabo-musulmane ${ }^{26}$. Cependant, si dans Version Soft et Sans titre 3 son visage donne l'impression de se faire graduellement détruire par l'interdit de la représentation à travers l'ajout d'accessoires ou par l'effacement de ses traits, la répétition de son visage dans ses différentes œuvres symbolise plutôt un processus de résilience. Benohoud crée ainsi des « corps suspects $^{27}$ " qui dérangent, à travers lesquels il se donne le droit d'exister en tant qu'individu, mais aussi en tant que sujet artistique et politique.

\section{Mehdi-Georges Lahlou: la question des genres de l'Occident à l'Orient}

En comparaison à la démarche de Benohoud, les autoportraits du Franco-Marocain MehdiGeorges Lahlou sont plutôt des « corps attentats $^{28}$ " où l'artiste s'attaque à plusieurs fronts, allant des tabous de la religion musulmane aux excès de la civilisation occidentale jusqu'à la
27 FENNANE Abedelghani, « La photographie, son corps et sa société ". Site Web de Hicham Benohoud. [En ligne], 2010, p. $9<$ http://www.hichambenohoud.com/benohoud/ index.php?option=com_content $\&$ view $=$ article $\& i d=54>$, consulté le 10 avril 2017.

28 GIQUEL Pierre, "Jusqu'au bout ». Dans site Web de Mehdi-Georges Lahlou. [En ligne], (2011) 2p. <http://mehdigeorges-lahlou.e-monsite.com/medias/files/pierremono.pdf>, consulté le 10 avril 2017. 


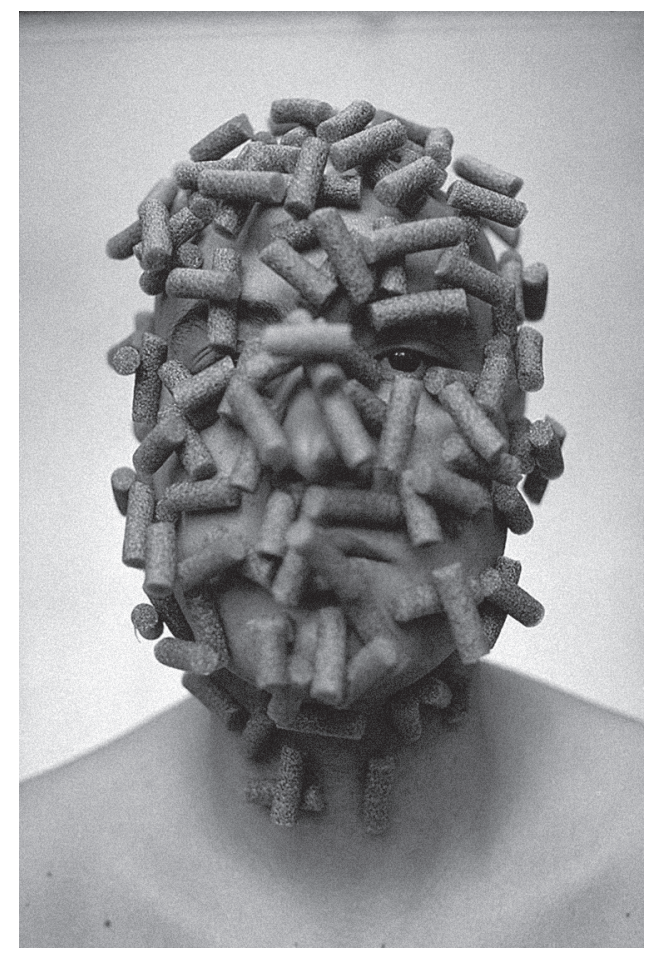

Fig. 1

Hicham Benohoud, Version Soft 09, 2003, photographie argentique, (C) Hicham Benohoud

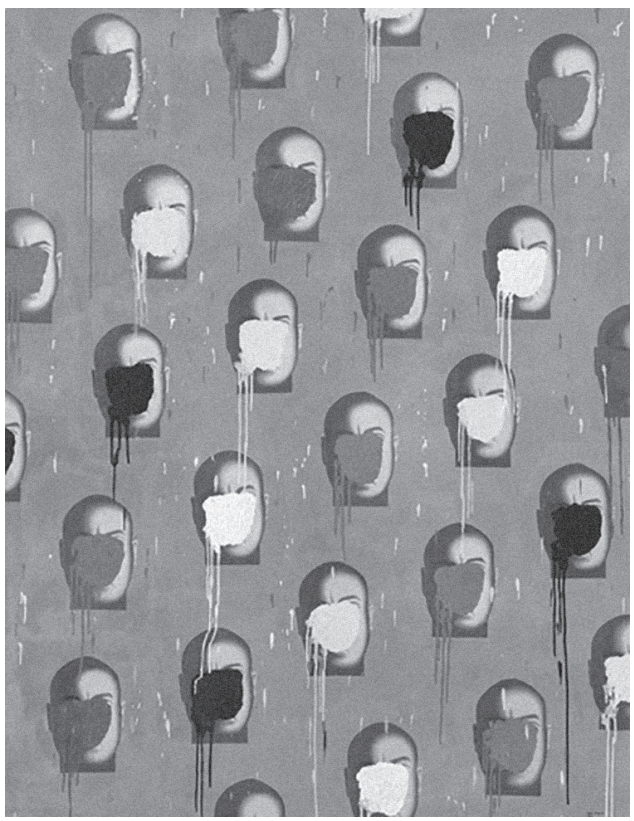

Fig. 2

Hicham Benohoud, Sans-titre 3, 2008, peinture à l'huile, $140 \times 180 \mathrm{~cm}$, () Hicham Benohoud 
question des genres. Se qualifiant d'artiste de "l'interstice ", entre l'Orient et l'Occident, Mehdi-Georges Lahlou brise les clichés entourant, par exemple, l'homosexualité, la féminité et, plus largement, les traditions musulmanes et chrétiennes dans le but d'évacuer toute tentative de catégorisation simpliste. S'attaquer à tous les stéréotypes sans distinction ni préférence s'avère un moyen pour contrer ce double emprisonnement que ressent l'artiste devant les multiples sentiments d'appartenances composant son individualité. Se positionnant contre toute essence réductrice de sa personne, MehdiGeorges Lahlou choisit le paradoxe en brouillant les pistes identitaires.

L'artiste utilise particulièrement la stratégie du travestissement pour s'éloigner de toute catégorie fermée, mais aussi pour expérimenter de nouvelles avenues du Soi. Ses œuvres s'inscrivent dans une rhétorique de l'entre-deux, où l'on peut certes déceler les facettes de l'identité et du genre de l'artiste, mais où l'on en entrevoit d'autres grâce, par exemple, à l'usage d'accessoires. Dans l'œuvre Portrait de famille, l'artiste emprunte l'apparence d'une femme musulmane voilée. En lien avec le titre de l'œuvre, à travers un jeu de symétrie, les cinq représentations de Mehdi-Georges Lahlou donnent l'impression de personnifier des membres d'une même famille. Jouant avec les ambiguiités, l'artiste y détourne le voile, un symbole de la femme musulmane, tout en assumant sa barbe, un symbole de maturité masculine, mais aussi de sagesse en Islam. La présence de cette barbe permet aussi de neutraliser l'image stéréotypée de l'homme à la sexualité ambiguë, traditionnellement considéré comme un être imberbe dans les cultures maghrébo-musulmanes ${ }^{29}$.

L'artiste utilise la même stratégie de travestissement dans l'œuvre Portrait selon Molinier... Neshat. Dans cette œuvre sous le mode du

29 CHEBEL Malek. Le corps en Islam. Paris: Puf, 2013 (1984), p. 64.

30 DABASHI Hamid, «The Gun and the Gaze: Shirin Neshat's Photography ". Dans le catalogue de la série Women Of Allah, Milan: Marco Noire Editore, 1997, p. 3. trompe-l'œil, le visage barbu de Mehdi-Georges Lahlou, dissimulé par de la calligraphie arabe, est entouré de deux mains féminines gainées donnant l'impression à l'observateur de regarder la représentation d'une femme voilée.

L'œuvre fait avant tout référence au photomontage intitulé Portrait d'Hanel Koeck, réalisé par l'artiste français Pierre Molinier en 1968. Dans cette photographie, Pierre Molinier voulait représenter différentes transgressions du genre masculin et féminin.L'esthétique de l'œuvre de Mehdi-Georges Lahlou en noir et blanc, la présence de la femme voilée et la calligraphie arabe sur le visage sont aussi une référence évidente à la célèbre série Women of Allah (1997) par l'artiste iranienne Shirin Neshat. Dans cette série d'autoportraits photographiques, Shirin Neshat inscrit sur son visage des extraits des poèmes de Forough Farrokhzad (1935-1967), une poétesse iranienne qui remettait en question les genres dans la religion musulmane. Si les œuvres de Neshat s'attaquent à une vision patriarcale de la femme musulmane, elles abordent aussi le problème du colonialisme. Tel que le précise avec justesse l'écrivain Hamid Dabashi: "The veil from behind which she looks back is not just the patriarchal imposition as a guard against modernity but is equally the colonial gaze which by exporting that modernity turned her into an object of desire, an odalisque of dangerous sensuality ${ }^{30}$ ". Mentionnons aussi l'importance de la présence des mains dans la démarche de Neshat, un symbole de puissance, mais aussi de protection (main de Fatma) dans les cultures maghrébo-musulmanes ${ }^{31}$. Dans la série Women of Allah, on voit l'artiste tendre les mains, mais aussi tenir un fusil ou un pistolet, déconstruisant ainsi l'image de la femme musulmane passive, incapable de se protéger des assauts du colonialisme et du patriarcat. Pour Neshat, les mains représentent un véhicule de pouvoir, car elles sont la seule partie du corps qui n'est pas voilée et qui permet aux femmes de travailler dans l'espace public. 
Dans l'autoportrait de Mehdi-Georges Lahlou, les mains gainées deviennent ainsi des organes de dénonciation où sont déconstruits les stéréotypes de la femme voilée musulmane passive, mais aussi de l'homme homosexuel qui aime se travestir.

Dans un autre registre, l'utilisation contemporaine de la calligraphie dans les œuvres de Mehdi-Georges Lahlou s'avère une stratégie polysémique pour détourner l'expérience figurative du portrait occidental, mais aussi pour réfléchir à l'interdiction de la représentation des vivants dans l'Islam. Mehdi-Georges Lahlou tente ainsi de réconcilier les tensions entre le portrait occidental et l'art islamique qui se développe sous des critères décoratifs. En inscrivant des mots en arabe dans l'œuvre Portrait selon Molinier... Neshat, Mehdi-Georges Lahlou détourne d'abord la calligraphie arabe en l'intégrant dans un espace pictural qui ne lui est pas réservé dans la tradition musulmane. Il remet cependant aussi en question l'histoire de l'art occidentale en y ajoutant de la calligraphie, un motif abstrait qui marque une nouveauté dans un genre caractérisé historiquement par la figuration.

\section{Zakaria Ramhani: les mots de l'autoportrait}

Dans une démarche comparable, les autoportraits réalisés avec le médium de la peinture de Zakaria Ramhani doivent leur caractère unique à une utilisation fascinante de la calligraphie arabe comme motif structurant des formes de son propre visage. Se consacrant inlassablement à la pratique de l'autoportrait depuis une quinzaine d'années, l'artiste allie le caractère ornemental de la calligraphie arabe en incluant des bribes de poésie ou des expressions populaires au portrait occidental. Ses peintures grand format où les visages se démultiplient proposent des réflexions sur l'Islam, l'actualité du monde arabe tout en faisant référence à la mémoire universelle de l'histoire de l'art.
L'influence de l'Islam orthodoxe et de l'aniconisme sur le monde artistique est impossible à évacuer de la démarche créative de Zakaria Ramhani. Les œuvres Faces of your Other et My Face is Word exposent des visages insaisissables qui oscillent entre l'abstraction et une figuration incertaine. Dans la série Faces of Your Other, l'artiste crée, à partir de visages miniatures, les différentes formes de son visage en grand format, à l'avant-plan de l'œuvre. Dans My Face is a Word, les visages miniatures participant à la construction du visage central de l'artiste ne sont pas des autoportraits, mais plutôt des portraits de plusieurs grands maîtres de l'histoire de l'art. On y reconnaît ainsi les fameux autoportraits de Van Gogh, Rembrandt, Dürer, Delacroix, Courbet, Bacon, Picasso et Kahlo. Ramhani fait aussi référence à la particularité de leurs démarches artistiques, puisque ces portraits miniatures sont peints selon le style respectif de ces différents artistes. L'historienne de l'art Monia Abdallah, dans la préface de l'exposition Mon visage est un mot, présentée à la Galerie l'atelier 21 (Casablanca, 2012), expliquait que sa production s'inscrivait en rapport au concept "d'ipséité ", tel qu'énoncé par le philosophe français Paul Ricour, présent dans toute identité. L'ipséité se définit comme une volonté de s'inscrire en continuité avec le temps, comme le fait ici Ramhani qui s'insère dans cette généalogie des grands autoportraitistes de l'histoire de l'art ${ }^{32}$.

Tel qu'il a été vu avec Portrait selon Molinier... Neshat par Mehdi-Georges Lahlou, la démarche artistique de Rahmani tente de réconcilier la peinture figurative liée à la tradition artistique occidentale avec son héritage arabo-musulman par des œuvres s'inscrivant hors des cadres de ces deux traditions. Ce rapport paradoxal entre l'image et le texte a comme influence première l'héritage de son père, un peintre marocain, qui portait en lui la culpabilité de peindre des représentations humaines malgré l'interdiction de figuration dans l'Islam. Depuis le début de sa carrière, Rahmani se joue de l'aniconisme en 


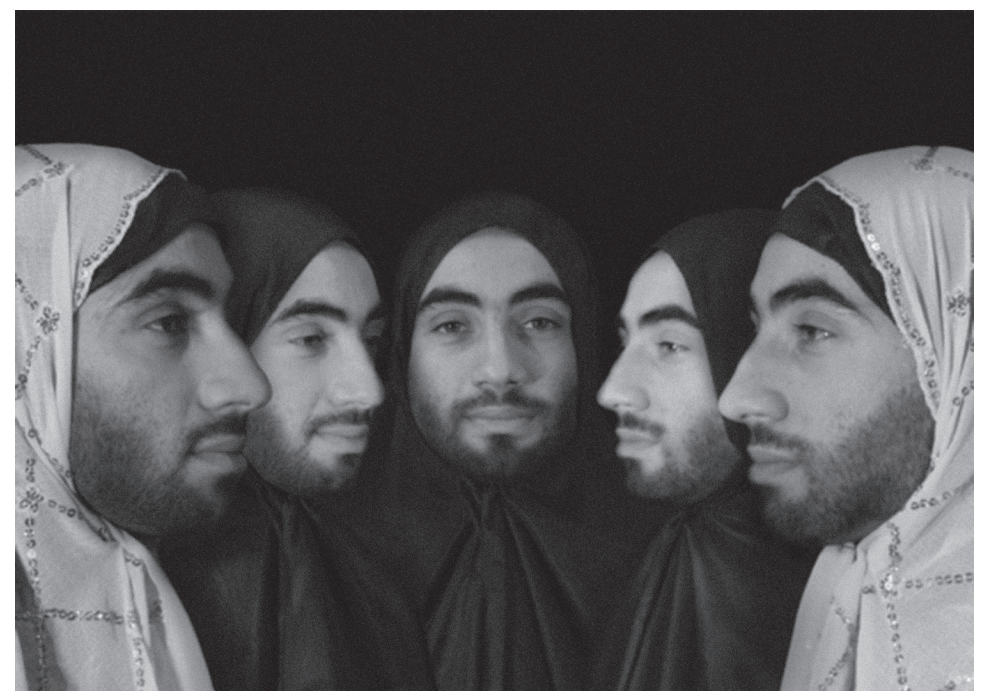

Fig. 3

Mehdi-Georges Lahlou, Portrait de famille, 2009, Photographie cprint, 16 x $25 \mathrm{~cm}$

(C) Mehdi-Georges Lahlou 


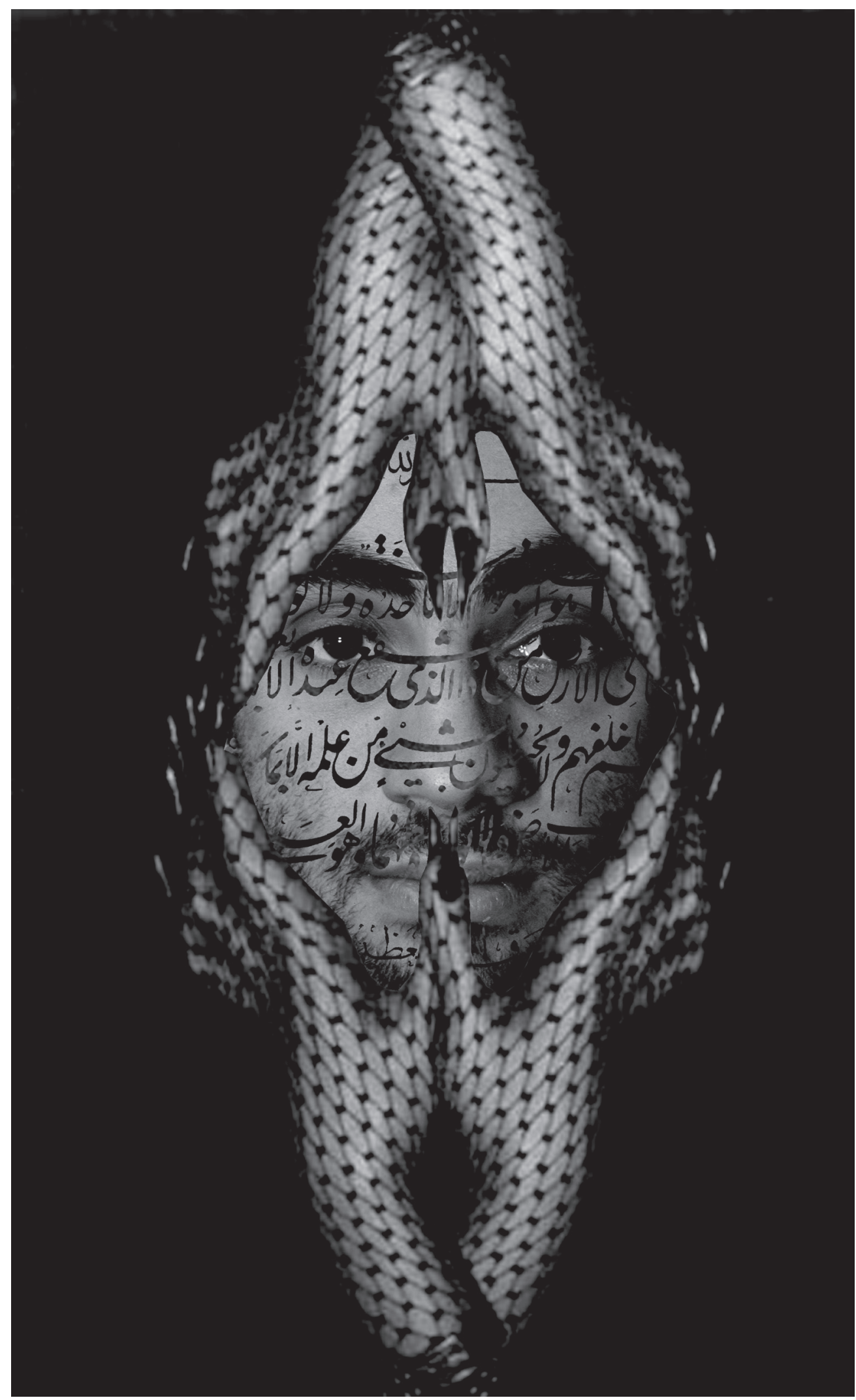




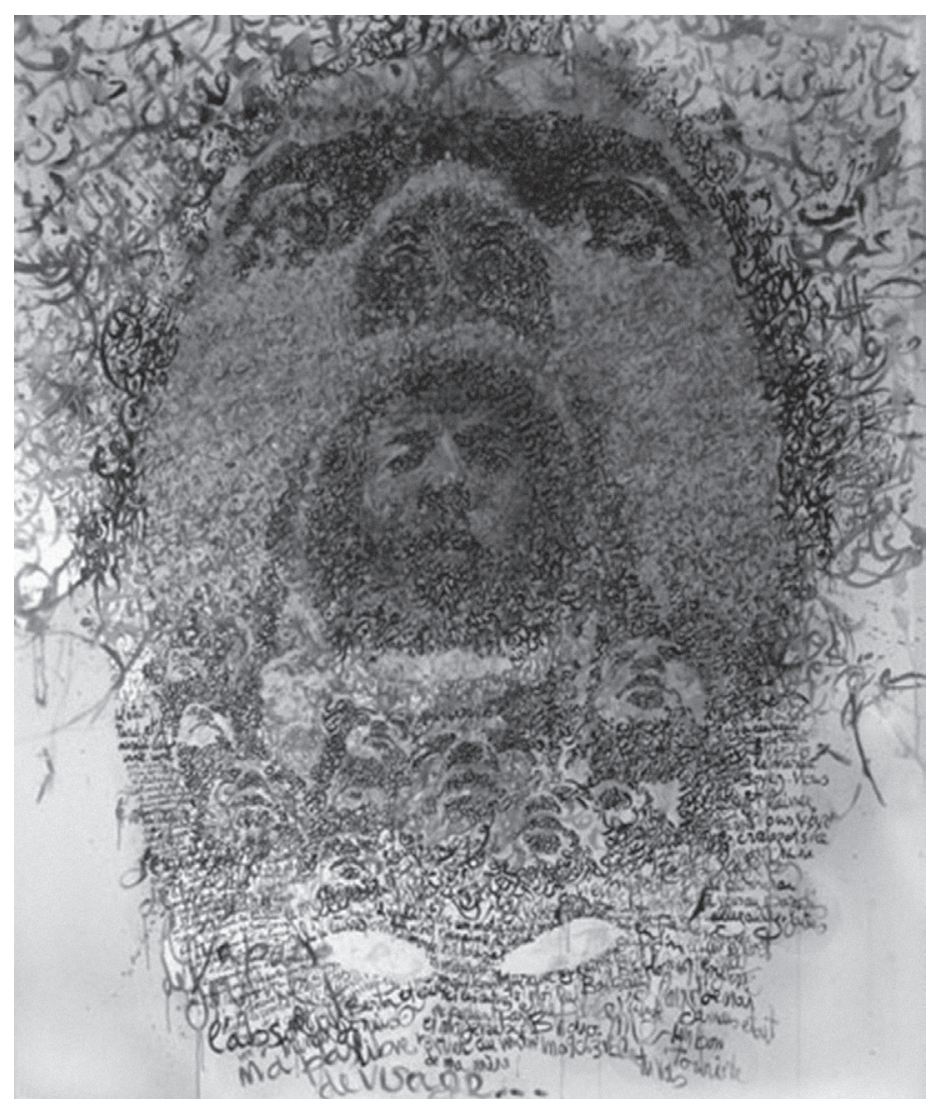

Fig. 5

Zakaria Ramhani, Face of your Other 07, 2010, huile sur toile, $240 \times 200 \mathrm{~cm}$, (C) Zakaria Ramhani

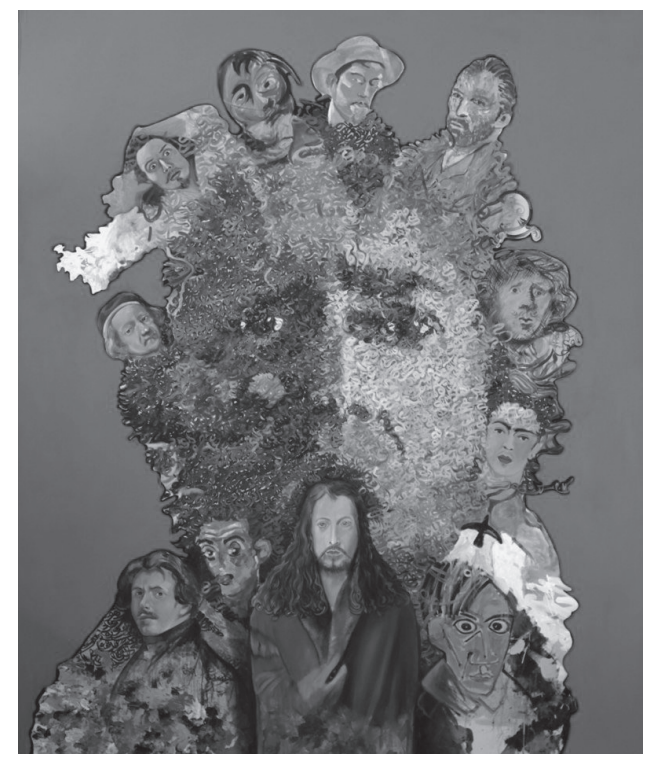

Fig. 6

Zakaria Ramhani, My Face is a Word, 2010, huile sur toile, 240 x 220 cm, (C) Zakaria Ramhani 
inventant un nouveau genre de représentation. En détournant la calligraphie arabe, une technique abstraite et ornementale utilisée traditionnellement par les artistes musulmans, il touche à la frontière de la figuration humaine en créant par des mots les formes de son visage. Si la calligraphie est lisible, la représentation du visage reste cependant très abstraite et se laisse deviner. S'interrogeant sur une possible cohabitation entre les mots et la forme dans l'art musulman, Ramhani ne réalise donc pas des peintures, mais il écrit plutôt des portraits ${ }^{33}$.

Au tournant des années 1980, Rasheed Araeen inscrivait lui aussi des mots en ourdou (sa langue maternelle) et en anglais sur ses autoportraits pour représenter les différents dilemmes qui construisent son sentiment d'appartenance en tant qu'artiste de la diaspora pakistanaise habitant au Royaume-Uni. S'inscrivant dans une démarche similaire, Ramhani mélange sa langue maternelle, l'arabe, au français et à l'anglais, les langues de l'Occident pour représenter les identités qui l'habitent. Ramhani refuse cependant de donner toutes les clés de lecture de ces œuvres à l'observateur. En effet, si l'allophone ne comprend pas les termes arabes inscrits sur ses toiles, l'arabophone non plus, puisque les mots se devinent difficilement dans ses œuvres où les différentes couches de peinture et de significations se juxtaposent l'une à l'autre.

\section{La pratique de l'autoportrait: une vision du corps locale}

À la suite de l'analyse de ce corpus d'œuvres polysémiques, on peut s'interroger sur les similitudes et les différences dans le traitement de l'autoportrait chez les trois artistes en lien avec leurs particularités culturelles. L'anthropologie visuelle soutient que la représentation du corps est le témoignage du contexte culturel propre à l'environnement de l'artiste. Par exemple, l'anthropologue David Le Breton énonce que la représentation du corps, et plus spécifiquement du visage, dans l'histoire de l'art est une construction identitaire d'ordre culturel, social et historique qui évolue au fil des siècles ${ }^{34}$.

L'anthropologue Malek Chebel développe plutôt la notion de civilisation du corps comme suit:

Nous entendons par le concept de civilisation du corps cette démarche réflexive qui considère non pas le corps pris isolément, mais surtout le corps tel qu'il se manifeste dans des prolongements symboliques, le corps répercuté sur le signe, sur la métaphore, sur le verbe et, en un sens, sur toute mentalisation offerte à l'expressivité populaire afin de se former en vue du projet social ${ }^{35}$.

\section{Dans l'ouvrage de référence Le corps en} Islam (1983), Chebel ancre ainsi les représentations du corps en rapport avec les particularités des civilisations du Maghreb. Pour l'auteur, le corps est un hiéroglyphe, soit un symbole ou une inscription à déchiffrer qui traduit l'histoire de l'individu, mais aussi celle collective d'une société. C'est dans cette optique que l'analyse des autoportraits de ces trois artistes a été considérée comme des manifestations d'une vision de la corporalité propre à l'imaginaire artistique du Maghreb.

Tel qu'il a été observé au fil des œuvres, le travail de trois artistes contemporains a comme principal point commun d'aborder le thème de l'interdiction de la représentation dans l'Islam. En effet, les motifs formels utilisés par les artistes pour s'autoreprésenter traduisent une réflexion actuelle, parfois critique, sur l'aniconisme qui marque historiquement les sociétés musulmanes. En effet, dans le cadre de la tradition prophétique des hadiths sunnites et chiites, il est interdit à l'homme de représenter les êtres vivants, puisque seul Dieu aurait le pouvoir de donner la vie. Tel que le précise le sociologue Abdelkébir Khatibi, le " [...] corps de l'homme, ses parties et l'âme qui les vivifie sont les reflets 
de la parole de Dieu transcrite sur un miroir vivant ${ }^{36}$ ". Les facteurs invoqués pour interdire les images humaines concernent principalement leur caractère impur rendant donc leur présence incompatible dans les lieux de prière. Après la démocratisation de la pratique photographique au milieu du $19^{\mathrm{e}}$ siècle, les images ont cependant envahi les domiciles et les villes des pays du Maghreb et du Moyen-Orient, complexifiant les interprétations par rapport à l'interdiction de la représentation ${ }^{37}$. Le travail des artistes témoigne justement qu'actuellement, l'image est toujours sujette à diverses tensions et débats selon les tendances plus strictes ou libérales des philosophies arabo-musulmanes. Les trois artistes font ainsi preuve d'une volonté de réinterpréter, voire d'outrepasser une représentation de type figurative par l'ajout d'artifices ou de matière plastique dans l'espace pictural dans le but de dissimuler à différents degrés les traits du visage. À ce titre, l'œuvre Portrait de famille (Mehdi-Georges Lahlou) utilise le travestissement et Version Soft (Hicham Benohoud), l'ajout d'accessoires pour détourner l'expérience figurative. Les œuvres Portrait selon Molinier... Nesthat (Mehdi-Georges Lahlou), My Face is a Word et Faces of your Other (Zakaria Ramhani) font appel à la calligraphie arabe pour dissimuler les traits des visages des artistes. Finalement, l'œuvre Sans-titre 3 (Hicham Benohoud) représente une destruction complète du visage de l'artiste sous le coup de l'interdiction de la représentation.

\section{Une conjonction des mondes}

Si le travail des trois artistes exprime les différentes sources construisant leur identité individuelle, il aborde aussi des thèmes d'ordre local et des problématiques plus larges, propres au contexte de la mondialisation. Jouant librement avec ces différentes références, ces artistes illustrent bien dans leur pratique les observa- tions suivantes de l'auteur Terri Smith: « Many artists working today imagine the physical conjunction of a number of different kinds of worlds: the intimate, personal sense of "my world"; the close neighborhood of the local; nearby worlds, then increasingly distant beyonds, until a sense of the world in general is reached ${ }^{38}$ ". Cette conjonction des différents mondes dans les œuvres présentées peut notamment être réfléchie selon le prisme de l'hybridité. Dans cette optique, l'auteur Anouar Ouyachchi affirme que la notion d'hybridité définirait une nouvelle philosophie contemporaine du corps dans le contexte de la production artistique marocaine qui permet " [...] d'aborder sous un éclairage plus complet la question de la tradition et des frontières culturelles dans un monde de plus en plus global qui tend à uniformiser les modèles d'identification ${ }^{39}$ ". Rappelons ici que pour Homi Bhabha, l'hybridité est un processus de négociation identitaire qui émerge dans des contextes sociaux et culturels s'inscrivant sous le signe de la rencontre avec l'Autre ${ }^{40}$. Les artistes marocains représentent ainsi la mobilité de leurs sentiments d'appartenances à travers des œuvres qui défont les frontières traditionnelles de l'histoire de l'art, mais aussi celles géographiques entre l'Orient et l'Occident. En effet, les différents points de tensions culturelles qui peuvent être lus dans les ouvres présentées témoignent de la situation de carrefour culturel et artistique du Maroc, situé entre l'Orient, l'Occident et l'Afrique. Les œuvres expriment aussi les déplacements qui marquent les parcours professionnels de ces artistes qu'ils soient marocains ou qu'ils proviennent d'une diaspora. Les autoportraits deviennent ainsi des espaces de transition, ou des tiers espaces, à travers lesquels peuvent être lues les différentes dynamiques qui caractérisent les spécificités locales des cultures de ces artistes, mais aussi la pluralité de leurs expériences du contexte de la mondialisation.
36 KHATIBI Abdelkébir. Euvres. Essais., Paris: La Différence, 2008, p. 91.

37 Voir à ce sujet: NAEF Silvia. Y a-t-il une "question de l'image " en Islam?, Paris: Téraèdre, 2004, 132 p.

38 SMITH Terri, Op.cit., p. 191.
39 OUYACHCHI Anouar. «De l'hybridité du corps dans l'art marocain ", revue Itinéraires, numéro spécial " Récits du corps au Maroc et au Japon ", n 3, 2011, p. 164. 40 Voir à ce sujet: Bhabha. Op.cit., p. 55-83. 
Par exemple, la démarche de Hicham Benohoud se positionne certes en rapport avec la situation actuelle marocaine, mais aussi en lien avec son expérience du monde de l'art en Europe. Tel que le précise Anouar Ouyachichi, ces visages s'inscrivent « $[\ldots]$ entre deux visions du corps appartenant à des cultures différentes: une vision occidentale où celui-ci est plus ou moins affirmé comme une propriété privée, et une autre arabo-musulmane qui en soumet les modalités et les conditions d'existence à la volonté du groupe ${ }^{41}$ ". Ces corps de l'entredeux expriment ainsi une vision où l'Orient serait la face négative de l'Occident et vice versa. En tant qu'artiste issu de la diaspora marocaine, Mehdi-Georges Lahlou, cet artiste de "l'interstice ", remet en question les catégorisations entourant les identités de genre en Islam, mais aussi les stéréotypes coloniaux et patriarcaux entourant les représentations de la femme musulmane. Zakaria Ramhani déconstruit la pureté des cultures maghrébo-musulmanes et occidentales à travers un usage original de la calligraphie arabe pour mettre de l'avant que les cultures sont des lieux de tension " $[\ldots]$ où le sujet est en concurrence avec un prétendu Autre $^{42}$ ». À travers ces œuvres, les trois artistes déconstruisent ainsi différentes catégorisations tout en prenant position contre les différents dogmes actuels à la fois religieux, social et sexuel qui homogénéisent les identités.

Cette proposition de lecture sous la forme d'une analyse entrecroisée de six autoportraits a porté un regard actuel sur la démarche de trois artistes marocains appartenant à l'un des nouveaux mondes de l'art propre à la pluralité des contextes de la mondialisation. Il a été vu que la formation et l'intégration institutionnelle des artistes se sont développées, en partie ou en totalité, sur le territoire euro-américains. Cette situation est notamment attribuable à un manque de soutien du monde de l'art marocain, obligeant ainsi les artistes à habiter ou à exposer en Occident pour ne pas être exclus des circuits internationaux. À travers ces œuvres, la représentation du corps véhiculée par les artistes canalise certains problèmes liés à la culture maghrébo-musulmane. En effet, ces créateurs critiquent sans hésitation la société marocaine en intégrant des références à l'aniconisme. Cependant, les autoportraits illustrent aussi une conjonction des mondes caractérisant l'expérience plurielle des artistes où se juxtaposent des préoccupations d'ordre individuel, local, mais aussi global. Ce regard croisé sur les mondes de l'autoportrait de ces trois artistes déconstruit ainsi l'hypothèse qu'il existerait un visage global de l'identité, mais démontre plutôt la possibilité d'une vision multiple de la corporalité au prisme des nouvelles narrativités du Soi du $21^{\mathrm{e}}$ siècle. 


\begin{abstract}
This article takes a contemporary look at the practice of self-portraiture by three artists from Morocco: Hicham Benohoud, Mehdi-Georges Lahlou and Zakaria Ramhani. The career trajectories of these creators illustrate the various dynamics of institutional opposition and integration mechanisms that belong to the new geographies of art, in the era of globalization. The article's goal is to investigate the manner in which individual, local and global concerns are expressed in their understanding of an imaginary projection of corporality. The works are studied through the lens of local characteristics particular to the artist's Maghreb-Muslim culture. In the first section, the artists' careers, in relation to their training and the places where they have exhibited their work, are presented in tandem with the paradoxes of globalization. It is thus specified that if the artists were trained primarily in the West or made their careers there to achieve international success, it can be attributed to a lack of support from the Moroccan artistic scene, but also to the difficult access to the international art world for artists coming from the art scenes of the Maghreb. In the second part, we postulate that the artists' self-portraits attest to the different issues related to MaghrebMuslim culture, such as the current socio-political situation in Morocco and the Islamic ban on representation. Lastly, we maintain that these self-portraits are a wider reflection of the varied concerns that transcend the borders of the Maghreb, as the artists reflect on the meeting of the Eastern and Western worlds.
\end{abstract}

